\title{
Villous adenoma of the bladder
}

\author{
J L Channer, J L Williams, L Henry
}

\begin{abstract}
A case of villous adenoma of the bladder associated with cystitis glandularis of intestinal type is described. Only three villous adenomas have been reported to date, of which two were also accompanied by cystitis glandularis. The lesion can be confused with polypoid hyperplasia in cystitis glandularis. Neutral mucins, acidic sulphomucins, and sialomucins were identified within the villous adenoma and adjacent areas of cystitis glandularis. It is suggested that the villous adenoma may form an intermediary stage in the development of some of the primary adenocarcinomas of the bladder arising in metaplastic intestinal mucosa.
\end{abstract}

$(\Im$ Clin Pathol 1993;46:450-452)

Villous adenomas arising in the bladder are rare tumours but have been described as isolated cases, ${ }^{1}$ or in association with cystitis glandularis. ${ }^{23}$ Some primary adenocarcino- mas of the bladder have also been described in patients with cystitis glandularis, although other adenocarcinomas are thought to arise from urachal remnants. ${ }^{4}$ Studies of the mucin histochemistry of cystitis glandularis and adenocarcinoma have been reported, ${ }^{56}$ but the mucin profile of villous adenomas of the bladder has not been examined.

\section{Case report}

A 73 year old man with persistent haematuria was referred for urological assessment. An ultrasound scan of the bladder suggested the presence of a carcinoma of the bladder. At cystoscopic examination, the bladder showed features of chronic retention, but there was also a papillary mass $2 \mathrm{~cm}$ from the vault. On bimanual palpation, the bladder wall felt oedematous. Bladder biopsy specimens showed noticeable inflammation with features of cystitis cystica and cystitis glandularis. No evidence of tumour was identified in this initial specimen but in view of the cystoscopic appearance, a further cystoscopy was per-
Department of Pathology. University of Sheffield Medical School

J L Channer

L Henry

Department of

Urology. Royal

Hallamshire Hospital, Sheffield

J L Williams

Correspondence to:

Dr J L Channer.

Department of

Histopathology, Northern

General Hospital,

Herries Road, Sheffield

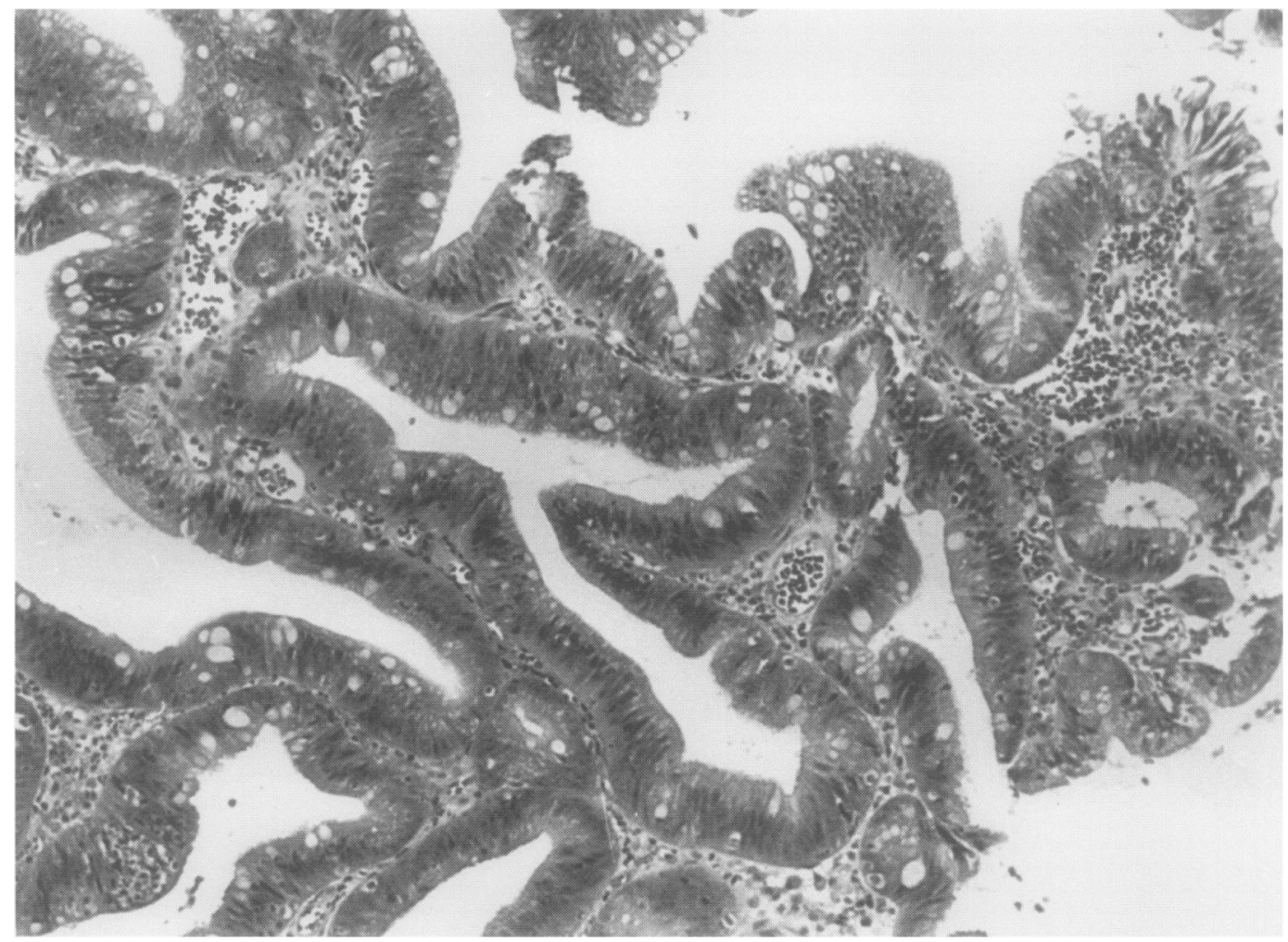

Villous adenoma of the bladder, consisting of papillary fronds covered by columnar epithelium with goblet cells, and showing nuclear crowding and stratification (haematoxylin and eosin). 
formed one month later and further bladder biopsy specimens were submitted for histological examination.

The biopsy specimens showed a range of changes, with prominent von Brunn's nests and areas of cystitis cystica and cystitis glandularis of both intestinal and non-intestinal type. Separate fragments of tissue consisting of long papillary fronds covered by intestinal type columnar epithelium, and including goblet cells, were also present (Figure) This epithelium also showed focal loss of nuclear polarity and crowding, suggestive of mild dysplasia. The appearances were of a villous adenoma arising within intestinal type cystitis glandularis. No stromal invasion was identified.

The tumour and adjacent cystitis glandularis were stained by combined alcian blue/periodic acid Schiff technique (ALB/ PAS), high iron diamine/ALB technique (HID/ALB), combined periodate-borohydride saponification-PAS technique (PB/ $\mathrm{KOH} / \mathrm{PAS}$ ), and Pascual's double silver impregnation technique. The adenoma and metaplastic epithelium showed similar staining characteristics: neutral mucins, acidic sulphomucins, and sialomucins were present in both the adenoma and metaplastic epithelium. O-acetylsialomucin, a type of mucin which was originally thought to be specific to the intestine, was also present. Occasional argyrophilic neuroendocrine cells were identified within the crypts, although these were not uniformly distributed. No Paneth cells were seen.

Eighteen months later the patient showed no evidence of tumour recurrence, although on regular review cystoscopy small areas of residual cystitis cystica persisted.

\section{Discussion}

Primary adenocarcinomas of the bladder are thought to arise from either urachal remnants or from intestinal metaplasia in cystitis glandularis. The presence of widespread cystitis glandularis of intestinal type is known to carry a significant risk for the subsequent development of adenocarcinoma. ${ }^{7}$ Villous adenomas are rare in comparison to adenocarcinomas, with only three individual cases having been reported so far. ${ }^{1-3}$ Two of these three, however, were also associated with the presence of cystitis glandularis. As so few cases have been reported, it is difficult to predict their behaviour, or assess their potential for malignant transformation. Morphologically, they resemble villous adenomas of the colon which are known to have the potential for malignant transformation. In other tissues-for example, the lung-malignant tumours can arise in a step-wise progression from epithelial metaplasia, to dysplasia, and then to invasive carcinoma. There may be a similar spectrum of changes in the bladder, progressing from metaplasia in cystitis glandularis, to dysplasia in a villous adenoma, and eventually to malignant change in an adenocarcinoma. In support of this theory, progres- sion of villous adenoma to carcinoma has been reported in the urethra. ${ }^{8}$

If there is a spectrum from intestinal metaplasia via adenoma to carcinoma in the bladder, it is difficult to explain the relative paucity of villous adenomas compared to adenocarcinomas. Progression to invasive tumour may occur rapidly within intestinaltype epithelium in the unnatural environment of the bladder, obscuring the original adenoma. Another explanation for the discrepancy is one of diagnosis. Occasionally extensive cystitis glandularis can show polypoid hyperplasia which can mimic a tumour both radiographically and cystoscopically. ${ }^{9}$ Therefore, the differential diagnosis of a papillary lesion in a patient with cystitis glandularis includes severe polypoid hyperplasia and a villous adenoma. Differentiation may indeed be impossible, particularly when the specimen is received as fragmented bladder biopsy specimens. However, a diagnosis of villous adenoma should be considered when there are both cytological and architectural differences from the adjacent metaplastic epithelium.

The mucin histochemical reactivity and other features of intestinal differentiation of adenocarcinomas of the bladder have been studied in an attempt to distinguish between morphologically similar tumours of urachal origin and those of metaplastic origin. ${ }^{5}$ In particular, o-acetylsialomucin, characteristic but not entirely specific to intestinal mucosa, was present in cystitis glandularis and an associated adenocarcinoma but was not present in an adenocarcinoma of urachal origin. If, as we suggest, the development of an adenoma is an intermediary stage in the formation of some adenocarcinomas, a similar mucin profile to that of metaplastic epithelium and adenocarcinoma would be expected in adenoma. As predicted, a range of mucins was identified within the adenoma and adjacent metaplastic epithelium similar to those reported in carcinomas, including the presence of o-acetylsialomucin. Another feature of intestinal differentiation, the presence of Paneth cells, was not found in the adenoma or metaplastic epithelium, although previously reported in adenocarcinoma of the bladder. ${ }^{10}$

Once villous adenoma has been diagnosed, it is important to exclude the presence of invasion. In the colon, breaching of the muscularis mucosae is regarded as the point at which a villous adenoma becomes an adenocarcinoma. In the bladder the superficial muscle coat is not as well defined and therefore the presence of invasion is more difficult to assess. Subjective features, such as the degree of dysplasia and surrounding stromal reaction, must be used. Compounding this problem is the inability to orientate fragments of tissue removed cystoscopically, compared with colonic resection specimens. As pathologists we therefore recommend adequate sampling of specimens showing the features of a villous adenoma, including the examination of multiple levels through the tissue as necessary, to exclude the 
possibility of invasion, and suggest that such patients should be reviewed regularly.

1 Makar N, Urquart AL. Adenoma of the urinary bladder. Br F Urol 1930;2:384-7.

2 Assor D. A villous tumour of the bladder. $f$ Urol 1978; 119:287-8.

3 Miller DC, Gang DL, Gavris V, Alroy J, Ucci AA, Parkhurst EC. Villous adenoma of the urinary bladder: a morphologic or biologic entity? Am $\mathcal{f}$ Clin Pathol 1983;79:728-31.

4 Thomas DG, Ward AM, Williams JL. A study of 52 cases of adenocarcinoma of the bladder. Br $\mathcal{F}$ Urol 1971; 43:4-15.

5 Wells M, Anderson K. Mucin histochemistry of cystitis glandularis and primary adenocarcinoma of the urinary bladder. Arch Pathol Lab Med 1985;109:59-61.

6 Alroy J, Roganovic D, Banner B, et al Primary adenocarcinoma of the human urinary bladder: histochemical, cinoma of the human urinary bladder: histochemical,
immunological and ultrastructural studies. Virchows immunological and ultrastructural studies. Virchows Arch(Pathol Anat) 1981;393:165-81.

7 Bullock PS, Thoni DE, Murphy WM. The significance of colonic mucosa (intestinal metaplasia) involving the urinary tract. Cancer 1987;59:2086-90.

8 Powell I, Cartwright H, Jano F. Villous adenoma and adenocarcinoma of the female urethra. Urology 1981;18: 612-14.

9 In: Young RH. Non-neoplastic epithelial abnormalities and tumor like lesions. Pathology of the urinary bladder. New York: Churchill Livingstone, 1989:1-640.

10 Pallesen G. Neoplastic Paneth cells in adenocarcinoma of the urinary bladder. Cancer 1981;47:1834-7. 